\title{
Estratégias para a Combinação de Classificadores Binários em Soluções Multiclasses
}

\author{
Ana Carolina Lorena ${ }^{1}$ \\ André C. P. L. F. de Carvalho ${ }^{2}$
}

\begin{abstract}
Resumo: Diversos problemas envolvem a classificação de dados em categorias, também denominadas classes. A partir de um conjunto de dados cujas classes são conhecidas, algoritmos de Aprendizado de Máquina podem ser utilizados na indução de um classificador capaz de predizer a classe de novos dados do mesmo domínio, realizando assim a discriminação desejada. Algumas técnicas de aprendizado são originalmente concebidas para a solução de problemas com apenas duas classes, também denominados binários. Entretanto, diversos problemas requerem a discriminação dos dados em mais que duas categorias ou classes. Neste artigo é apresentada uma revisão de estratégias para a generalização de técnicas de aprendizado binárias para a solução de problemas com mais que duas classes, intitulados multiclasses. O foco é em estratégias que decompõem o problema multiclasses original em múltiplos subproblemas binários, cujas saídas são combinadas na obtenção da classificação final.
\end{abstract}

\begin{abstract}
Several problems involve the classification of data into categories, also called classes. Given a dataset containing data whose classes are known, Machine Learning algorithms can be employed for the induction of a classifier able to predict the class of new data from the same domain, performing the desired discrimination. Some learning techniques are originally conceived for the solution of problems with only two classes, also named binary problems. However, several problems require the discrimination of examples into more than two categories or classes. This paper surveys strategies for the generalization of binary classifiers to problems with more than two classes, known as multiclass problems. The focus is on strategies that decompose the original multiclass problem into multiple binary subtasks, whose outputs are combined to obtain the final classification.
\end{abstract}

\section{Introdução}

Diversas técnicas de Aprendizado de Máquina (AM) [34] são originalmente formuladas para a solução de problemas de classificação binários. Entre elas pode-se citar as Má-

\footnotetext{
${ }^{1}$ Centro de Matemática, Computação e Cognição, Universidade Federal do ABC, Rua Catequese, 242, CEP 09090400 , Santo André, SP, ana. lorena@ufabc.edu.br

${ }^{2}$ Departamento de Ciências de Computação, Instituto de Ciências Matemáticas e de Computação, Universidade de São Paulo, Caixa Postal 668, CEP 13560-970, São Carlos, SP, andre@ icmc . usp.br
} 
quinas de Vetores de Suporte (SVMs, do Inglês Support Vector Machines) [6, 7, 48]. Muitos problemas de classificação, contudo, apresentam mais que duas classes.

A generalização de técnicas de classificação binária para problemas multiclasses pode ser realizada basicamente por meio de duas estratégias. A primeira consiste na combinação de preditores gerados em subproblemas binários, enquanto na segunda realiza-se adaptações nos algoritmos originais das técnicas consideradas. A extensão direta de um algoritmo binário a uma versão multiclasses nem sempre é possível ou fácil de realizar [36]. Para as SVMs, em particular, Hsu e Lin [15] observaram que a reformulação dessa técnica em versões multiclasses leva a algoritmos computacionalmente custosos. É comum recorrer-se então à alternativa de decompor o problema multiclasses em subproblemas binários, uma estratégia denominada decomposicional.

Segundo Moreira e Mayoraz [35], existe uma série de motivações para empregar estratégias decomposicionais na solução de problemas multiclasses. Além de haver técnicas cuja formulação é originalmente binária, alguns algoritmos não são adequados a problemas com um número elevado de classes ou apresentam dificuldades em lidar com grandes volumes de dados de treinamento. Mesmo que o algoritmo seja capaz de trabalhar com problemas de grande escala, o uso de um procedimento decomposicional pode reduzir a complexidade computacional envolvida na solução do problema total, por meio da divisão deste em subtarefas mais simples.

Pimenta [40] também aponta vantagens no uso da decomposição em problemas cujos erros de classificação das diferentes classes possuem pesos distintos. Pode-se assim gerar preditores binários de maneira a impor preferências para algumas classes. Esse autor menciona ainda o fato dos subproblemas de classificação gerados em uma decomposição serem independentes, podendo ser solucionados paralelamente.

O emprego de técnicas decomposicionais envolve dois passos. No primeiro realizase a divisão do problema multiclasses em subproblemas binários, determinando assim os classificadores binários a serem gerados. A segunda etapa pode ser denominada reconstrução e refere-se à forma como as saídas dos classificadores binários são combinadas na determinação da classe de um exemplo [32].

Neste artigo são apresentadas diversas estratégias decomposicionais. De forma geral, é possível agrupá-las em dois tipos: baseadas em matrizes de códigos e hierárquicas. Esses tipos são descritos nas seções 2 e 3, respectivamente. Na Seção 4 este artigo é concluído com algumas considerações finais. 


\section{Estratégias Baseadas em Matrizes de Códigos}

A abordagem de matrizes de códigos unifica uma série de técnicas decomposicionais [1]. Essas estratégias podem ser representadas de maneira geral por uma matriz de códigos M. As linhas dessa matriz contêm códigos que são atribuídos a cada classe. As colunas de $\mathbf{M}$ definem partições binárias das $k$ classes e correspondem aos rótulos que essas classes assumem na geração dos classificadores binários. Tem-se então uma matriz de dimensão $k x l$, em que $k$ é o número de classes do problema e $l$ representa o número de classificadores binários utilizados na solução multiclasses. Na Figura 1 é apresentado um exemplo de matriz de códigos na qual o número de classes $k$ é igual a quatro e o número de classificadores binários $l$ também é quatro. Abaixo dessa matriz de códigos são indicadas as partições binárias das classes realizadas por cada classificador binário representado em suas colunas.

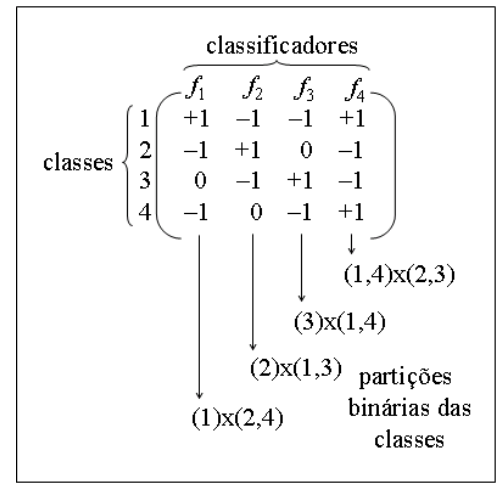

Figura 1. Exemplo de matriz de códigos para um problema com quatro classes

Cada elemento da matriz $\mathbf{M}$ assume valores em $\{-1,0,+1\}$. Um elemento $m_{i j}$ com valor +1 indica que a classe correspondente à linha $i$ assume rótulo positivo na indução do classificador $f_{j}$. $\mathrm{O}$ valor -1 designa um rótulo negativo e um valor 0 indica que os dados da classe $i$ não participam do processo de indução do classificador $f_{j}$. Classificadores binários são então treinados de forma a aprender os rótulos representados nas colunas de $\mathbf{M}$.

\subsection{Etapa de Reconstrução}

Um novo dado $\mathrm{x}$ pode ser classificado avaliando-se as predições dos $l$ classificadores, que geram um vetor $\mathbf{f}(\mathbf{x})$ de tamanho $l$ na forma $\mathbf{f}(\mathbf{x})=\left(f_{1}(\mathbf{x}), \ldots, f_{l}(\mathbf{x})\right)$. Esse vetor é então comparado às linhas de $\mathbf{M}$. $\mathrm{O}$ dado é atribuído à classe cuja linha de $\mathrm{M}$ é mais próxima de $\mathbf{f}(\mathbf{x})$ de acordo com alguma medida. Esse processo de reconstrução também é referenciado como decodificação [37]. Seja $\mathbf{m}_{q}$ a $q$-ésima linha de $\mathbf{M}$, a qual apresenta o código referente 
à classe $q$. O processo de decodificação equivale a computar a Equação 1, em que $f(\mathbf{x})$ representa o classificador multiclasses final e $d$ denota uma função de decodificação.

$$
f(\mathbf{x})=\underset{1 \leqslant q \leqslant k}{\arg \min }\left(d\left(\mathbf{m}_{q}, \mathbf{f}(\mathbf{x})\right)\right)
$$

Caso mais de um código se encontre mais próximo a $\mathbf{f}(\mathbf{x})$, tem-se a ocorrência de uma classificação desconhecida. Escolhe-se então uma das classes envolvidas no empate aleatoriamente ou com o uso de alguma informação a priori [44].

Existem diversas funções de decodificação que podem ser utilizadas na integração dos classificadores binários na Equação 1 [37]. A mais simples é a de Hamming, que conta o número de ocorrências diferentes entre o vetor $\mathbf{f}(\mathbf{x})$ e cada um dos códigos. Essa função pode ser visualizada na Equação 2. Um rótulo 0 contribui com $\frac{1}{2}$ na computação da soma apresentada. A função descrita equivale à distância de Hamming caso $\mathbf{M}$ possua unicamente os rótulos +1 e -1 [36].

$$
d_{H}\left(\mathbf{m}_{q}, \mathbf{f}(\mathbf{x})\right)=\sum_{i=1}^{l} \frac{1-\operatorname{sgn}\left(m_{q i} * f_{i}(\mathbf{x})\right)}{2}
$$

Existem várias outras funções de decodificação que podem ser empregadas na integração dos classificadores binários [37, 53]. Em um trabalho recente, Passerini et al. [37] apresentaram uma função baseada em probabilidades condicionais para as SVMs, em que, a partir das margens de classificação, se obtém probabilidades de que o dado pertença a cada classe.

\subsection{Etapa de Decomposição}

As descrições realizadas anteriormente referiam-se à etapa de reconstrução das saídas multiclasses a partir dos preditores binários representados na matriz $\mathrm{M}$. Na decomposição do problema, diversas alternativas podem ser empregadas. A decomposição mais compacta de um problema com $k$ classes pode ser realizada com o uso de $l=\left\lceil\log _{2}(k)\right\rceil$ classificadores binários [32]. Um exemplo de matriz compacta para um problema com quatro classes é apresentado na Figura 2a.

O número total de diferentes preditores binários para um problema com $k$ classes é $0,5\left(3^{k}+1\right)-2^{k}$, considerando que $f=-f$, ou seja, que a inversão das classes positivas e negativas produz o mesmo classificador [32]. Desses, $2^{k-1}-1$ incluem todas as classes simultaneamente, ou seja, possuem somente rótulos $+1 \mathrm{e}-1$, sem o elemento 0 [32]. Para quatro classes, tem-se nesse caso a matriz representada na Figura $2 b$. 


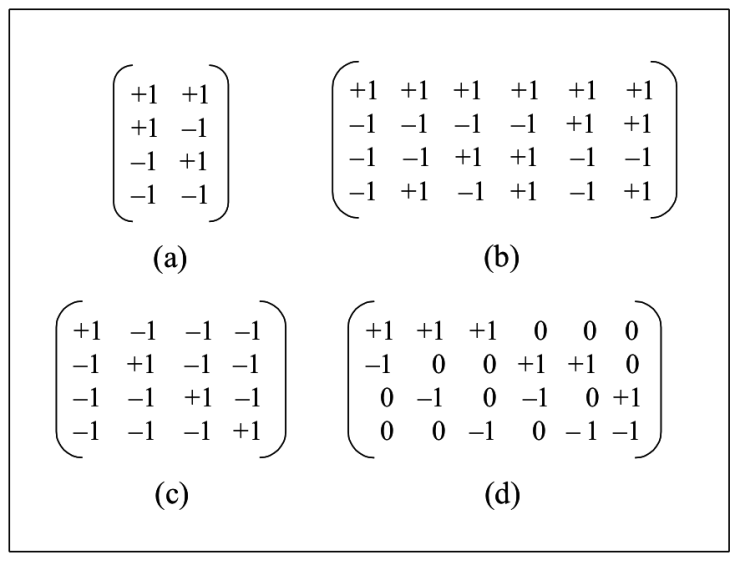

Figura 2. Matrizes de diferentes decomposições para um problema com quatro classes

Entre as estratégias decomposicionais mais comuns encontradas na literatura estão a um-contra-todos (one-against-all - OAA) [51], a todos-contra-todos (all-against-all, também denominada one-against-one - OAO) [13, 18] e a baseada em códigos de correção de erros [9], que são descritas a seguir.

Na estratégia OAA, dado um problema com $k$ classes, $k$ classificadores binários $f_{i}(\mathbf{x})$ são gerados. Cada um desses preditores é treinado de forma a distinguir uma classe $i$ das demais. A representação dessa técnica é dada por uma matriz de dimensão $k x k$, na qual os elementos da diagonal possuem o valor +1 e os demais, o valor -1 . Uma matriz do tipo OAA para um problema de quatro classes é apresentada na Figura 2c. Usualmente, na integração dos classificadores OAA, escolhe-se a classe correspondente ao classificador que produz a maior saída [43].

A decomposição OAA pode apresentar desvantagens quando a proporção de exemplos de uma classe é muito pequena em relação a do conjunto formado pelos dados das outras classes. Esse tipo de desbalanceamento pode dificultar a indução de um preditor que apresente bom desempenho no reconhecimento da classe considerada.

Na decomposição OAO, dadas $k$ classes, $\frac{k(k-1)}{2}$ classificadores binários são gerados. Cada um deles é responsável por diferenciar um par de classes $(i, j)$, em que $i \neq j$. A matriz de códigos nesse caso possui então dimensão $k \mathrm{x} \frac{k(k-1)}{2}$ e cada coluna corresponde a um classificador binário para um par de classes. Em uma coluna representando o par $(i, j)$, o valor do elemento correspondente à linha $i$ é +1 e o valor do membro correspondente a $j$ é igual a -1 . Todos os outros elementos da coluna possuem o valor 0 , indicando que os 
dados de outras classes não participam do processo de indução do classificador. Na Figura $2 \mathrm{~d}$ tem-se uma matriz OAO para um problema com quatro classes.

Embora o número de classificadores gerados na decomposição OAO seja da ordem de $k^{2}$, o treinamento de cada um deles envolve dados de apenas duas classes. Com isso, mesmo com um número elevado de classes, o tempo total despendido na geração dos preditores geralmente não é grande.

A integração usual dos classificadores gerados na estratégia OAO é realizada por meio de um esquema de votação por maioria [20]. Dado um novo exemplo x, cada classificador fornece um voto em sua classe preferida. O resultado é então dado pela classe que recebeu mais votos. A implementação dessa solução corresponde ao uso da função de Hamming sobre a matriz de códigos descrita anteriormente.

Um problema apontado na decomposição OAO é que a resposta de um preditor para um par de classes $(i, j)$ na realidade não fornece informação alguma quando o exemplo não pertence às classes $i$ ou $j$ [2].

Em uma estratégia decomposicional alternativa, Dietterich e Bariki [9] propuseram o uso de códigos de correção de erros para representar as $k$ classes de um problema. Para tal, os autores sugerem que o valor do número de classificadores $l$ seja maior que o número mínimo necessário para diferenciar cada classe unicamente. Os bits adicionais introduzem uma redundância na codificação das classes e têm a utilidade de prover ao sistema a capacidade de se recuperar de eventuais erros cometidos por alguns preditores no processo de classificação de um novo exemplo. Por esse motivo, essa técnica possui a denominação de decomposição por códigos de correção de erros (ECOC, do Inglês Error Correcting Output Codes). As matrizes de códigos nesse caso assumem valores em $\{-1,+1\}$ e utiliza-se a função de Hamming na decodificação das saídas dos classificadores.

Com esse procedimento de correção de erros, Dietterich e Bariki [9] sugerem que a solução de um problema multiclasses por uma técnica de AM seja vista como uma tarefa de comunicação. Dado um novo exemplo, a sua classe correta é transmitida por meio de um canal, o qual é constituído de seus atributos, dos dados de treinamento e do algoritmo de aprendizado. Devido a erros que podem estar presentes nos atributos do exemplo, nos dados de treinamento utilizados ou em falhas no processo de aprendizado, a informação pode ser corrompida. Para prover ao sistema a capacidade de se recuperar desses erros de transmissão, a classe é codificada por um código de correção de erros e cada bit do mesmo é transmitido separadamente, ou seja, por execuções separadas do algoritmo de aprendizado.

Para possibilitar a correção de erros, Dietterich e Bariki [9] sugerem que os códigos das classes contidos em $\mathbf{M}$ sejam bem separados segundo a distância de Hamming. Sendo $d_{l}$ a distância mínima entre qualquer par de linhas de $\mathbf{M}$, então o classificador multiclasses final é capaz de corrigir ao menos $\left\lfloor\frac{d_{l}-1}{2}\right\rfloor$ bits incorretos em uma predição. Como segundo 
a função de decodificação de Hamming cada bit incorreto implica em se afastar em uma unidade do código da classe correta, cometendo $\left\lfloor\frac{d_{l}-1}{2}\right\rfloor$ erros, o código mais próximo ainda será o correto [9]. Segundo esse princípio, a codificação OAA é incapaz de se recuperar de qualquer erro, uma vez que nesta $d_{l}=2$.

Além disso, na construção de bons códigos de correção de erros, deve-se estimular também que os erros dos classificadores binários gerados sejam não correlacionados. Exigese então a separação entre as colunas de $\mathbf{M}$, ou seja, a distância de Hamming entre cada par de colunas deve ser grande. Se no algoritmo de aprendizado a inversão das classes positivas e negativas produz o mesmo classificador (ou seja, $f=-f$ ), então deve-se também fazer com que a distância de Hamming entre cada coluna e o complemento das outras seja grande.

Com base nessas observações, Dietterich e Bariki [9] propuseram quatro técnicas para o desenvolvimento de matrizes de códigos com boa capacidade de correção de erros. A escolha de cada uma delas é determinada pelo número de classes do problema. Para $k \leqslant 7$, esses autores recomendam o uso de um código exaustivo, que consiste da combinação de $2^{k-1}-1$ classificadores binários unicamente com rótulos $+1 \mathrm{e}-1$, conforme ilustrado na Figura $2 \mathrm{~b}$ para um problema com quatro classes. A distância $d_{l}$ em uma matriz gerada pelo método exaustivo é de $2^{k-2}$. Se $8 \leqslant k \leqslant 11$, é aplicado um método que seleciona colunas do código exaustivo. Para $k>11$, tem-se duas opções: um método baseado no algoritmo hillclimbing e a geração de códigos $\mathrm{BCH}$ [3], os quais provêm da teoria de códigos de correção de erros em comunicação.

Em um trabalho recente, Pimenta [40] propôs um algoritmo para o desenvolvimento de ECOCs que, usados com SVMs, apresentaram resultados compatíveis ou superiores aos de estratégias decomposicionais tradicionais. Nele é proposta uma função para a avaliação da qualidade de ECOCs segundo suas propriedades de correção de erros. Um algoritmo iterativo de perseguição é então utilizado na construção dos ECOCs. Esse algoritmo adiciona ou retira colunas de um ECOC inicial, procurando maximizar a sua qualidade. Pimenta [40] também apresentou um método para a determinação do número de colunas no ECOC, por meio do exame de uma função de avaliação baseada no número de erros corrigíveis por ECOCs de diferentes tamanhos.

Em Allwein et al. [1] é apontado que, apesar dos códigos gerados pelo ECOC possuírem boa propriedade de correção, vários dos subproblemas binários criados podem ser difíceis de aprender. Por esse motivo, as técnicas mais simples OAA e OAO têm apresentado resultados comparáveis ou superiores ao ECOC em várias aplicações [1, 43].

Uma crítica comum às estratégias OAA, OAO e ECOC é que todas realizam a decomposição do problema a priori, sem levar em consideração as propriedades e as características de cada aplicação $[1,2,8,19,32,31,44]$. Essa foi a motivação de diversos trabalhos para a proposição de técnicas para encontrar matrizes de códigos adaptadas à solução de cada 
problema multiclasses.

Entre eles, em [25, 29, 22] apresentou-se uma técnica que utiliza Algoritmos Genéticos (AGs) [33] para encontrar combinações de classificadores binários adequadas à solução de cada problema multiclasses considerado. Os AGs foram utilizados na obtenção de matrizes de códigos relacionadas a cada aplicação, adaptando-as de acordo com o desempenho obtido na solução do problema multiclasses.

Duas versões de AGs foram implementadas. Ambas manipulam matrizes de códigos, procurando minimizar o seu erro na solução multiclasses e, ao mesmo tempo, reduzir o número de preditores binários contidos nas mesmas. Além disso, procurou-se evitar a presença de classificadores idênticos em uma mesma matriz de códigos, fato que representaria o uso de preditores iguais em uma mesma decomposição. O primeiro AG, denominado lexicográfico, impõe uma ordem nos dois primeiros objetivos, privilegiando a minimização do erro sobre a do número de preditores binários. Evitar a ocorrência de preditores binários idênticos foi considerada uma restrição do problema. O segundo AG, denominado SPEA2 (Strength Pareto Evolutionary Algorithm 2) [54], lida com os três objetivos descritos simultaneamente, por meio do uso do conceito de dominância de Pareto.

Experimentalmente, usando SVMs na geração dos preditores binários, o AG lexicográfico se mostrou mais adequado. Os principais benefícios verificados na aplicação desse AG se deram na obtenção de decomposições com desempenho comparável ao de estratégias tradicionais, porém mais simples que elas, por necessitar de menos classificadores binários.

Experimentos preliminares empregando esses mesmos AGs para determinar também parâmetros diferenciados para cada SVM binária em uma decomposição apresentaram resultados animadores [22]. A busca por valores de parâmetros diferenciados para cada SVM é um problema difícil de ser solucionado empiricamente. Por esse motivo, o uso de um procedimento de busca mais sofisticado, que realiza essa procura de forma automática, pode ser uma alternativa promissora. Apesar dos bons resultados iniciais, experimentos adicionais são necessários.

Em [32] foi apresentado um algoritmo iterativo que adiciona classificadores binários a uma matriz de códigos considerados pertinentes de acordo com a distribuição dos dados das classes. Com isso, a construção dos códigos leva em consideração as características dos dados. Ele procura ainda maximizar a distância entre as linhas e as colunas dessa matriz, segundo os princípios para a obtenção de códigos com propriedade de correção de erros. A maior contribuição nos resultados alcançados por esse procedimento foi a obtenção de classificadores multiclasses mais simples que os do ECOC.

Em [2] apresentou-se um algoritmo que determina matrizes de códigos com a utilização de uma Rede Neural Artificial (RNA) do tipo Perceptron Multicamadas [4, 14]. Essa RNA também define os parâmetros de classificadores lineares que são utilizados na solução 
dos subproblemas binários contidos na matriz. Por meio do treinamento da RNA, otimiza-se conjuntamente os parâmetros dos classificadores lineares e a matriz de códigos utilizada na decomposição do problema multiclasses. Comparado a empregar as decomposições ECOC, OAA e OAO com classificadores lineares, o método proposto obteve alguns ganhos em termos de taxa de acerto.

Dekel e Singer [8] introduziram um algoritmo denominado Bunching, que adapta uma matriz de códigos para cada problema multiclasses em seu processo de aprendizado. Esse algoritmo mapeia os dados de treinamento e os seus rótulos para um novo espaço. A matriz de mapeamento dos rótulos corresponde à matriz de códigos do problema. No novo espaço, procura-se minimizar a distância entre os dados e os seus rótulos. $\mathrm{O}$ algoritmo de Bunching parte de uma matriz de códigos inicial, que é iterativamente adaptada ao problema. Contudo, os elementos das matrizes de códigos neste trabalho são probabilísticas, ou seja, seus elementos são contínuos e não discretos. Empiricamente, os autores verificaram bons resultados na adaptação de matrizes dos tipos OAA e aleatórias, utilizando regressores logísticos como classificadores binários.

Em [44] a adaptação de matrizes de códigos a cada aplicação multiclasses foi formulada como um problema de otimização no qual os códigos, e também pesos para as funções binárias utilizadas no aprendizado das decomposições, são determinados em conjunto. Maximiza-se a diferença entre a distância do vetor de previsões dos classificadores binários em relação ao código da classe correta e a distância desse mesmo vetor ao segundo código mais próximo. Alguns resultados experimentais iniciais apresentados indicaram uma vantagem da aplicação desse método com Árvores de Decisão (ADs) [42] em relação ao uso direto dessa técnica de aprendizado na solução multiclasses.

\section{Estratégias Hierárquicas}

Uma maneira alternativa de solucionar um problema multiclasses com preditores binários pode ser conduzida com sua decomposição de uma forma hierárquica. Em diversos domínios, existem hierarquias naturais entre as classes. Um exemplo é a categorização de textos, na qual algumas classes de textos podem ser agrupadas em sub-tópicos, os quais por sua vez também podem ser reunidos em tópicos principais. De forma geral, a introdução de uma hierarquia em uma aplicação multiclasses pode reduzir a complexidade de sua solução. A idéia é realizar inicialmente discriminações mais gerais, as quais são refinadas sucessivamente até a obtenção da classificação final.

Na Figura 3 são apresentados dois exemplos de classificadores multiclasses hierárquicos utilizados na solução de um problema com seis classes [47]. O classificador da Figura 3a possui uma estrutura de árvore direcionada binária, em que exatamente uma aresta chega a cada nó e no máximo duas arestas partem dos mesmos. Na Figura $3 b$ tem-se uma estru- 
tura mais geral, de um grafo direcionado acíclico, em que mais de uma aresta pode apontar para um mesmo nó. Segundo a definição, as árvores direcionadas binárias são um tipo de grafo direcionado acíclico. Porém, para facilitar as exposições realizadas nesta seção, essas estruturas serão tratadas como tipos distintos. Em ambas as estruturas, cada nó interno corresponde a um classificador binário que distingue dois subconjuntos de classes, enquanto os nós terminais, denominados folhas, representam as classes individuais. Iniciando pelo nó raiz, as classes são particionadas recursivamente em dois subconjuntos, até que todos os subconjuntos gerados contenham apenas uma classe.

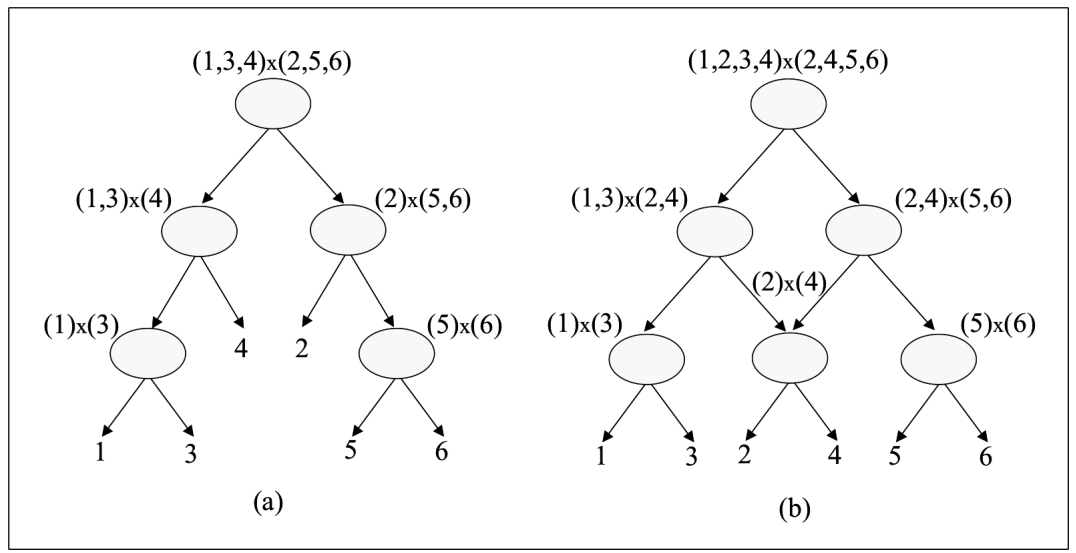

Figura 3. Classificadores hierárquicos para um problema com seis classes [47]

Na previsão da classe de um novo exemplo, este é inicialmente submetido à raiz da hierarquia. Baseado na decisão desse preditor, um novo nó é visitado. Esse processo é iterado até que uma folha seja atingida, na qual a classe é determinada. Esse é o procedimento de reconstrução comumente empregado na obtenção de classificações com o uso de uma estrutura hierárquica. Os preditores e ramificações percorridos na classificação de um exemplo formam um caminho no classificador hierárquico comumente referenciado como caminho de avaliação do exemplo.

Em geral, pode-se afirmar que as estratégias hierárquicas apresentam tempos menores de predição do que as baseadas em matrizes de códigos anteriormente discutidas. Isso ocorre porque, na classificação de um dado, apenas uma parte dos preditores binários são consultados, enquanto a decodificação das matrizes de códigos necessita das saídas de todos classificadores na decomposição.

Deve-se observar que os preditores contidos nas estratégias hierárquicas também podem ser representados por uma matriz de códigos. Entretanto, a forma de integração deles é 
dependente de cada dado e não envolve a consulta de todos os preditores binários, como na aplicação das funções de decodificação apresentadas na Seção 2. No caso das estratégias hierárquicas, a decodificação aplicada é conhecida por eliminação [17] e opera iterativamente. Inicialmente todos os classificadores binários (colunas da matriz) são considerados ativos. Em uma determinada iteração, um deles é consultado. As classes que "perdem" são eliminadas, assim como os classificadores binários relacionados a elas. Esse processo é repetido até que um único classificador binário reste, provendo a classificação final.

Para a obtenção das hierarquias, que equivale à etapa de decomposição do problema, várias estratégias podem ser empregadas. Algumas delas são discutidas a seguir. Inicialmente são apresentadas abordagens com estrutura de grafos direcionados acíclicos, seguindo de estruturas de árvores direcionadas binárias.

\subsection{Grafos Direcionados Acíclicos}

Em [41], é sugerido que os classificadores produzidos pela decomposição OAO sejam dispostos em um grafo de decisão direcionado acíclico (Decision Directed Acyclic Graph DDAG). Logo, cada nó do grafo corresponde a um preditor binário para um par de classes. Essa estratégia será referenciada como DDAG neste documento.

Na Figura 4 é ilustrado um exemplo de DDAG para um problema com quatro classes. A cada nó está associada uma lista de classes. Inicialmente, todas as classes são candidatas e o nó raiz distingue as classes 1 e 4, equivalentes à primeira e à última classes da lista, respectivamente. A partir de cada resultado dessa partição, uma das classes é eliminada da lista e um novo nó é definido, separando as classes inicial e final da nova lista formada. Esse processo é iterado até a obtenção das folhas, que equivalem a listas com apenas uma classe.

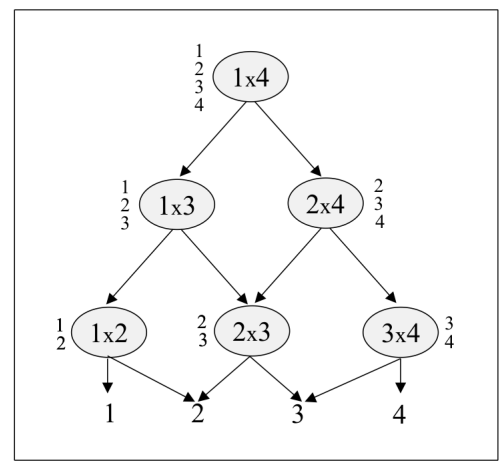

Figura 4. Exemplo de DDAG para um problema com quatro classes [41] 
Pode-se verificar que, na classificação de um novo exemplo com essa estrutura, $k-1$ classificadores binários são avaliados. Logo, essa estrutura acelera a fase de predição da abordagem OAO.

Uma desvantagem do DDAG, apontada em [16], é sua dependência em relação à sequiência de classificadores binários nos nós do grafo. Essa característica afeta a sua confiabilidade, uma vez que diferentes permutações de nós no grafo podem produzir resultados distintos. Considere, por exemplo, o problema com três classes ilustrado na Figura 5. Podese recorrer a hiperplanos para separar os pares de classes, os quais encontram-se indicados pelas fronteiras traçadas entre as classes. Na região sombreada encontram-se pontos para os quais, dependendo da estrutura de DDAG utilizada, classificações diferentes são produzidas. Esse fato também encontra-se ilustrado na mesma figura, a qual apresenta os três possíveis DDAGs para um problema com três classes. Verifica-se que a classificação de um dado na região sombreada por cada um deles produz saídas distintas. Logo, a estrutura do DDAG tem influência na saída final produzida.

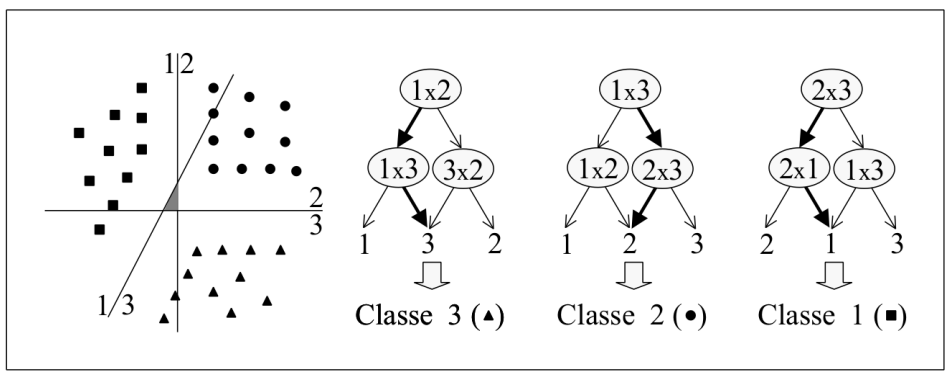

Figura 5. Classificação na região sombreada difere de acordo com a estrutura do DDAG

Outra deficiência do DDAG apontada em [16] é que, dependendo da posição da classe correta no grafo, o número de avaliações com esta classe é desnecessariamente grande, resultando em um alto erro cumulativo. Se a classe correta de um exemplo encontra-se no nó raiz, por exemplo, ela será testada $k-1$ vezes contra as outras para a geração da saída desejada. Se há uma probabilidade de $1 \%$ de erro em cada nó, isto irá causar uma taxa de erro cumulativo de $1-(0.99)^{k-1}$, que se torna crítica especialmente para valores altos de $k$. Logo, se na predição do rótulo de um dado a classe correta deste encontra-se em níveis próximos da raiz, essa classe é exposta a um maior risco de ser rejeitada erroneamente.

Esses fatores motivaram Kijsirikul e Ussivakul [16] a desenvolverem uma nova estratégia hierárquica para combinar as saídas produzidas por classificadores obtidos pela decomposição OAO. A nova estrutura, denominada DAG Adaptável (ADAG, do Inglês Adaptive Directed Acyclic Graph), corresponde a um DAG com estrutura reversa. Na Figura 6 é apre- 
sentado um exemplo de ADAG para um problema com oito classes. O ADAG tem $k-1$ nós, cada qual correspondendo a um classificador binário para um par de classes. A primeira camada tem $\left\lceil\frac{k}{2}\right\rceil$ nós, seguidos por $\left\lceil\frac{k}{2^{2}}\right\rceil$ na segunda camada e assim por diante, até que uma camada com um único nó é atingida, a qual produz a saída final.

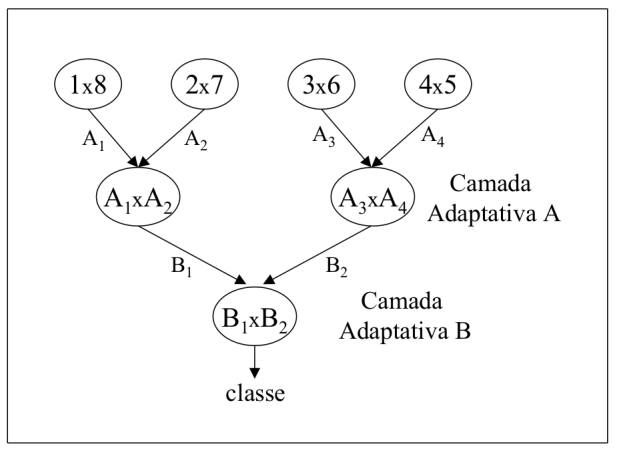

Figura 6. Exemplo de ADAG para problema com oito classes [16]

Para a classificação de um novo dado utilizando o ADAG, inicialmente ele é avaliado por todos os classificadores do primeiro nível, os quais diferenciam pares distintos de todas as classes. No caso do número de classes ser ímpar, um dos nós contém apenas uma classe, a qual é diretamente passada para o segundo nível. Cada um dos preditores binários produz como saída sua classe preferida. Baseado nas escolhas do nível anterior, os classificadores das próximas camadas são determinados. A cada passagem de nível, o número de classes candidatas é reduzido pela metade. Como no DDAG, $k-1$ nós são avaliados em cada predição. Porém, a classe correta é testada contra outras classes $\left\lceil\log _{2} k\right\rceil$ ou menos vezes, número menor que no DDAG, que pode requerer até $k-1$ avaliações com essa classe.

Utilizando essa estrutura, o ADAG minimiza a ocorrência de erros cumulativos, especialmente em problemas com um número de classes elevado. Embora o ADAG também tenha demonstrado menor dependência que o DDAG em relação à ordem dos classificadores binários no grafo, ainda ocorrem diferenças de desempenho entre estruturas distintas. Logo, a definição dos classificadores que compõem o primeiro nível do ADAG afeta o seu resultado.

Alguns trabalhos investigaram heurísticas para determinar as estruturas de ADAGs ou DDAGs $[10,38,39,50]$. O número possível de diferentes DDAGs para um problema com $k$ classes é $\frac{k !}{2}$ e de ADAGs é $\frac{k !}{2\lfloor k / 2\rfloor}$. Na obtenção desses valores, classificadores para os pares de classes $(i, j)$ são considerados equivalentes àqueles para os pares $(j, i)$, ou seja, considera-se $f=-f$.

Em [39] foi proposta uma modificação no algoritmo original do ADAG, denominada 
Reordering ADAG (RADAG), que permite adequar a estrutura do ADAG ao problema multiclasses sendo solucionado. O algoritmo é voltado ao uso de SVMs e os classificadores binários de cada nível do RADAG são definidos ordenando os pares de classes $(i, j)$ tal que a soma dos $\left\|\mathbf{w}_{i j}\right\|$ seja minimizada, em que cada $\left\|\mathbf{w}_{i j}\right\|$ é determinado no processo de treinamento de uma SVM na decomposição. A cada classificação de um exemplo, os preditores binários nos níveis do RADAG vão sendo determinados de acordo com essa regra. Logo, o RADAG mantém a característica do ADAG de adaptar a estrutura do grafo de acordo com cada dado de teste, porém utiliza-se a informação dos $\left\|\mathbf{w}_{i j}\right\|$ nesse processo. Em [38] pareiase as classes de acordo com a capacidade de generalização dos classificadores binários, estimada por um limite de erro no caso das SVMs. Procura-se parear as classes de forma a minimizar a soma desse erro. Os autores reportaram resultados com o uso do procedimento descrito comparáveis ou superiores aos de ADAGs gerados aleatoriamente e do OAO com votação por maioria.

Outro trabalho que propõe uma heurística para a determinação da estrutura dos DAGs é [50]. Os autores sugerem que os classificadores binários com maior capacidade de generalização sejam colocados em níveis superiores do DDAG. Em [10], o mesmo tipo de heurística é utilizado na definição da estrutura de DDAGs. Takahashi e Abe [50] reportam que essa heurística levou à obtenção de estruturas de DDAGs com desempenho semelhante à média de estruturas geradas de maneira aleatória, utilizando SVMs na geração dos classificadores binários. Takahashi e Abe [50] também apontam que o algoritmo proposto pode ser facilmente estendido para os ADAGs.

Em [26], AGs foram utilizados na busca da ordem dos nós em um DAG (DDAG ou ADAG), baseado em seu desempenho na solução do problema multiclasses. Esse problema foi reduzido a um problema de permutação, em que uma lista de classes é ordenada, e foi então solucionado a partir de analogias com o uso de AGs na solução de problemas de permutação, como o do caixeiro viajante. Utilizando SVMs na indução dos classificadores binários, verificou-se poucas variações de desempenho entre estruturas de DAGs distintas, o que fez com que as soluções encontradas pelos AGs atingissem resultados, em termos de taxa de acerto, similares aos de estruturas geradas aleatoriamente.

\section{2 Árvores Direcionadas Binárias}

Diversos trabalhos empregam uma estrutura em forma de árvore direcionada binária na obtenção do classificador multiclasses hierárquico. As árvores possuem $k-1$ classificadores binários e, portanto, envolvem o treinamento de $k-1$ preditores, menor número entre todas as estratégias decomposicionais principais mencionadas anteriormente. Além disso, os nós de níveis inferiores envolvem cada vez menos classes e, portanto, menos dados de treinamento para os classificadores binários correspondentes. Na fase de teste, no melhor caso, dependendo da estrutura da árvore, é possível classificar o exemplo já no primeiro nó. No 
pior caso, os $k-1$ classificadores devem ser consultados. Logo, a fase de teste pode ser acelerada nessa estrutura frente às anteriormente discutidas.

Para um problema com $k \geqslant 3$ classes, existem $\prod_{i=3}^{k} 2 i-3$ estruturas de árvores distintas [11]. Duas possíveis árvores para um problema com quatro classes são ilustradas na Figura 7. Como no DDAG e no ADAG, a estrutura da árvore, ou seja, que classificadores são dispostos na árvore e onde eles se encontram, influencia o seu resultado. Os trabalhos nessa área diferenciam então no processo de obtenção das partições binárias das classes em cada nó da árvore e, conseqüentemente, na determinação de sua estrutura. Todos aplicam algum critério recursivamente sobre subconjuntos de classes, particionando-os em dois até que eles possuam apenas uma classe.

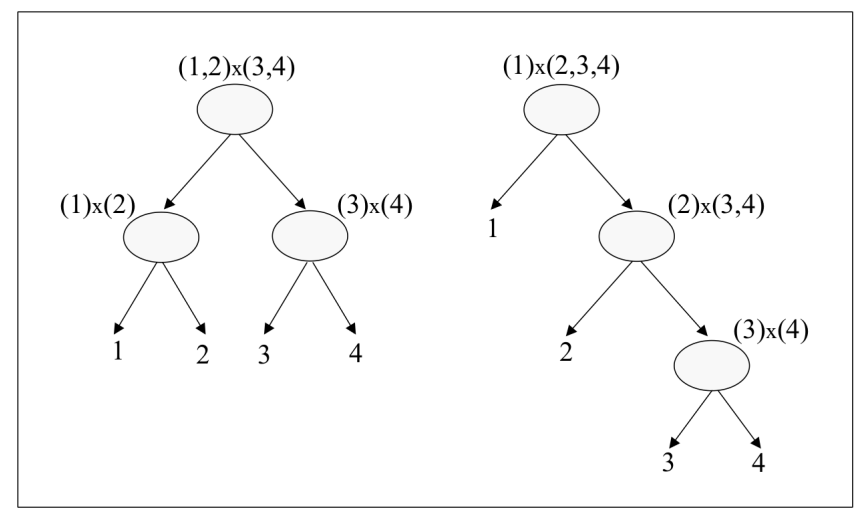

Figura 7. Duas árvores para um problema com quatro classes [11]

Na determinação da estrutura de árvores multiclasses, Takahashi e Abe [49] propõem que nós próximos à raiz particionem classes mais facilmente separáveis. Para medir a separabilidade, foram utilizadas a distância Euclidiana entre os centros das classes e informações dos dados incorretamente classificados por um preditor que os separa de acordo com a sua distância de Malahanobis aos centros das classes. Os métodos propostos foram comparados por Takahashi e Abe [49] em um experimento abreviado e apresentaram resultados similares.

Em [46] e [47] utilizou-se o conceito de classes de confusão na definição das partições binárias de classes na hierarquia. Uma classe de confusão representa um subconjunto de classes para as quais há uma grande semelhança entre os dados, tal que mesmo uma pequena quantidade de ruídos pode levar a classificações errôneas [47]. Para quantificar a confusão entre as classes, empregou-se o algoritmo de agrupamento k-médias [34], com k $=2$. Avaliando o desempenho da árvore obtida por esse método na classificação de dígitos manuscritos, 
Schwenker e Palm [47] verificaram que a sua taxa de acerto foi similar a das estratégias OAA e OAO com votação por maioria.

Em [52] foram sugeridos três métodos para a partição binária das classes nos nós de árvores multiclasses. O primeiro agrupa classes com centróides similares utilizando o algoritmo k-médias. O segundo divide as classes de acordo com a distância média de seus dados à origem. $\mathrm{O}$ terceiro divide as classes em subconjuntos tal que a diferença entre o número de dados neles seja mínima. O terceiro critério foi efetivamente utilizado nos experimentos e se mostrou vantajoso principalmente se as classes encontram-se desbalanceadas, ou seja, apresentam grandes diferenças na quantidade de dados.

Em [21], as partições binárias das classes foram definidas por um algoritmo de agrupamento hierárquico. Utilizou-se a distância média entre os dados das classes no processo de agrupamento. Os resultados experimentais obtidos foram similares aos das estratégias OAA, OAO com votação por maioria e DDAG.

Frank e Krammer [11] propõem que diversas árvores multiclasses sejam combinadas em um comitê, como alternativa a determinar uma única estrutura. Os autores demostraram empiricamente a viabilidade de sua proposta em relação ao uso das estratégias OAA, OAO, ECOC e da solução direta do problema multiclasses, empregando ADs e classificadores de regressão logística na indução dos preditores binários.

Em [22], dois algoritmos foram propostos para definir as partições binárias de classes em uma árvore. Realizou-se adaptações no algoritmo de Kruskal, o qual obtém árvores geradoras mínimas a partir de grafos ponderados. Essas alterações permitiram que os preditores binários a serem contidos nas árvores multiclasses fossem determinados automaticamente a partir de informações coletadas dos dados de cada aplicação multiclasses. Ambos os algoritmos realizam um agrupamento hierárquico das classes de acordo com a sua similaridade. Cada agrupamento realizado define diretamente uma partição binária. Um grafo que pondera a relação entre as classes é utilizado no processo de agrupamento. Esse grafo possui $k$ vértices, que representam as $k$ classes do problema, e $\frac{k(k-1)}{2}$ arestas entre todos os pares de vértices. Os pesos são calculados de acordo com informações extraídas do conjunto de dados do problema multiclasses. Desta forma, a estrutura da árvore é definida de acordo com as características e propriedades dos dados de cada aplicação multiclasses [27, 28, 30]. Utilizando SVMs na indução dos classificadores binários, as taxas de acerto obtidas pelas árvores foram similares às de estratégias tradicionais, utilizando, entretanto, menos classificadores binários na solução multiclasses. 


\section{Considerações Finais}

Embora algumas técnicas de aprendizado sejam originalmente formuladas para a solução de problemas de classificação binários, o seu uso pode ser estendido a aplicações multiclasses. Em geral dois tipos de estratégias podem ser empregados nesse processo: decomposicionais e diretas. As decomposicionais dividem o problema total em múltiplos subproblemas binários, cujas saídas são combinadas na obtenção da previsão multiclasses. Nas estratégias diretas, reformula-se o algoritmo original da técnica de aprendizado em uma versão multiclasses. Entretanto, esse procedimento nem sempre é simples de ser realizado. Este artigo revisou as principais estratégias decomposicionais da literatura. Outras técnicas também podem ser consultadas em [23, 22].

Ainda com relação às estratégias decomposicionais, foram apresentadas duas abordagens para a representação e a agregação dos classificadores binários produzidos: baseada em matrizes de códigos e hierárquicas. Porém, há também trabalhos que combinam as saídas dos preditores binários com o uso de outro classificador. Esse classificador, que pode ser produzido por outra técnica de aprendizado distinta da utilizada na obtenção dos preditores binários, é treinado de forma a ponderar as saídas dos classificadores binários na previsão multiclasses. Entre os trabalhos que fizeram uso desse tipo de estratégia, pode-se mencionar: $[24,31,45]$.

Como uma possível linha trabalho futuro, pode-se citar a investigação de quais características dos dados de cada aplicação tornam uma estratégia multiclasses mais adequada à sua solução. Pode-se utilizar, por exemplo, estratégias de meta-aprendizado nesse processo $[5,12]$. Uma vez que os desempenhos de diferentes estratégias multiclasses podem apresentar algumas variações nas classes, a combinação de um subconjunto delas também pode acarretar em benefícios, caso haja complementariedades entre os seus resultados. Uma outra possibilidade é atribuir pesos aos preditores binários nas decomposições, o que é realizado, por exemplo, em [8]. Um preditor binário considerado mais importante por algum critério pode assim possuir maior peso na decisão final.

O levantamento bibliográfico realizado evidencia que as pesquisas sobre a geração de preditores multiclasses a partir de técnicas de classificação binárias têm sido intensas, o que também pode ser constatado pela presença de várias publicações recentes na área. De forma geral, cada publicação aponta as vantagens de uma técnica particular sobre outras. $\mathrm{Na}$ prática, entretanto, pode-se dizer que cada técnica tem vantagens e também deficiências e seus resultados dependem da aplicação sendo considerada e também do bias da técnica de AM empregada na indução dos classificadores binários. 


\section{Referências}

[1] E. L. Allwein, R. E. Shapire, and Y. Singer. Reducing multiclass to binary: a unifying approach for magin classifiers. In Proceedings of the 17th International Conference on Machine Learning, pages 9-16. Morgan Kaufmann, 2000.

[2] E. Alpaydin and E. Mayoraz. Learning error-correcting output codes from data. In Proceedings of the 9th International Conference on Neural Networks, pages 743-748, 1999.

[3] R. C. Boser and D. K. Ray-Chaudhuri. On a class of error-correcting binary group codes. Information and Control, 3:68-79, 1960.

[4] A. Braga, A. C. P. L. F. Carvalho, and T. B. Ludermir. Redes Neurais Artificiais: Teoria e Aplicações. Editora LTC, 2000.

[5] P. Brazdil, J. Gama, and B. Henery. Characterizing the applicability of classification algorithms using meta level learning. In Proceedings of the European Conference on Machine Learning, volume 784 of Lecture Notes in Artificial Intelligence, pages 83102. Springer-Verlag, 1994.

[6] C. Cortes and V. N. Vapnik. Support vector networks. Machine Learning, 20(3):273296, 1995.

[7] N. Cristianini and J. Shawe-Taylor. An Introduction to Support Vector Machines and other kernel-based learning methods. Cambridge University Press, 2000.

[8] O. Dekel and Y. Singer. Multiclass learning by probabilistic embeddings. In Advances in Neural Information Processing Systems, volume 15, pages 945-952. MIT Press, 2003.

[9] T. G. Dietterich and G. Bariki. Solving multiclass learning problems via error-correcting output codes. Journal of Artificial Intelligence Research, 2:263-286, 1995.

[10] J. Feng, Y. Yang, and J. Fan. Fuzzy multi-class SVM classifier based on optimal directed acyclic graph using in similar handwritten chinese characters recognition. In J. Wang, X. Liao, and Z. Yi, editors, Proceedings of the International Symposium on Neural Networks, volume 3496 of Lecture Notes in Computer Science, pages 875-880. Springer-Verlag, 2005.

[11] E. Frank and S. Kramer. Ensembles of nested dichotomies for multi-class problems. In Proceedings of the 21st International Conference on Machine Learning, pages 305312. ACM Press, 2004.

[12] C. Giraud-Carrier, R. Vilalta, and P. Brazdil. Introduction to the special issue on metalearning. Machine Learning, 54:187-193, 2004. 
[13] T. Hastie and R. Tibshirani. Classification by pairwise coupling. The Annals of Statistics, 2:451-471, 1998.

[14] S. Haykin. Neural Networks - A Compreensive Foundation. Prentice-Hall, New Jersey, 2nd edition, 1999.

[15] C.-W. Hsu and C.-J. Lin. A comparison of methods for multi-class support vector machines. IEEE Transactions on Neural Networks, 13(2):415-425, March 2002.

[16] B. Kijsirikul and N. Ussivakul. Multiclass support vector machines using adaptive directed acyclic graph. In Proceedings of International Joint Conference on Neural Networks (IJCNN 2002), pages 980-985, 2002.

[17] A. Klautau, N. Jevtić, and A. Orlistky. On nearest-neighbor error-correcting output codes with application to all-pairs multiclass support vector machines. Journal of Machine Learning Research, 4:1-15, 2003.

[18] S. Knerr, L. Personnaz, and G. Dreyfus. Single-layer learning revisited: a stepwise procedure for building and training a neural network, pages 41-50. Springer-Verlag, 1990.

[19] J. Ko, E. Kim, and H. Byun. Improved n-division output coding for multiclass learning problems. In Proceedings of the 17th International Conference on Pattern Reognition, pages 470-473. IEEE Computer Society Press, 2004.

[20] U. Kre $\beta$ el. Pairwise classification and support vector machines. In B. Schölkopf, C. J. C. Burges, and A. J. Smola, editors, Advances in Kernel Methods - Support Vector Learning, pages 185-208. MIT Press, 1999.

[21] H. Lei and V. Govindaraju. Half-against-half multi-class support vector machines. In Nikunj C. Oza, Robi Polikar, Josef Kittler, and Fabio Roli, editors, Proceedings of the 6th International Workshop on Multiple Classifier Systems, volume 3541 of Lecture Notes in Computer Science, pages 156-164. Springer-Verlag, 2005.

[22] A. C. Lorena. Investigação de estratégias para a geração de máquinas de vetores de suporte multiclasses. PhD thesis, Instituto de Ciências Matemáticas e de Computação, Universidade de São Paulo, São Carlos. Disponível em: http: / / www . teses . usp . $\mathrm{br} /$ teses/disponiveis/55/55134/tde-26052006-111406/, 2006.

[23] A. C. Lorena and A. C. P. L. F. Carvalho. Revisão de técnicas para geração de classificadores de margens largas multiclasses. Technical Report 221, Instituto de Ciências Matemáticas e de Computação, Universidade de São Paulo, São Carlos. Disponível em: ftp://ftp.icmc.usp.br/pub/BIBLIOTECA/rel_tec/RT_221.ps, Novembro 2003. 
[24] A. C. Lorena and A. C. P. L. F. Carvalho. Comparing techniques for multiclass classification with binary SVM predictors. In Raul Monroy, Gustavo Arroyo-Figueroa, Luis Enrique Sucar, and Juan Humberto Sossa Azuela, editors, MICAI 2004: Advances in Artificial Intelligence, Third Mexican International Conference on Artificial Intelligence, Mexico City, Mexico, volume 2972 of Lecture Notes in Artificial Intelligence, pages 272-281. Springer-Verlag, 2004.

[25] A. C. Lorena and A. C. P. L. F. Carvalho. A GA/SVM approach for multiclass classification applied to protein structural class prediction. In A. Barros, A. Araújo, H. C. Yehia, and R. Teixeira, editors, Proceedings of the VIII Brazilian Symposium on Neural Networks, CD. UFMA, IEEE Computer Society and SBC, 2004.

[26] A. C. Lorena and A. C. P. L. F. Carvalho. An hybrid GA/SVM approach for multiclass classification with directed acyclic graphs. In A. L. C. Bazzan and S. Labidi, editors, Advances in Artificial Intelligence - SBIA 2004, 17th Brazilian Symposium on Artificial Intelligence, São Luis, Maranhão, Brazil, volume 3171 of Lecture Notes in Artificial Intelligence, pages 366-375. Springer-Verlag, 2004.

[27] A. C. Lorena and A. C. P. L. F. Carvalho. Minimum spanning trees in hierarchical multiclass support vector machines generation. In M. Ali and F. Esposito, editors, Innovations in Applied Artificial Intelligence: 18th International Conference on Industrial and Engineering Applications of Artificial Intelligence and Expert Systems, IEA/AIE 2005, Bari, Italy, volume 3533 of Lecture Notes in Artificial Intelligence, pages 422431. Springer-Verlag, 2005.

[28] A. C. Lorena and A. C. P. L. F. Carvalho. Protein cellular localization with multiclass support vector machines and decision trees. In J.C. Setubal and S. Verjovski-Almeida, editors, Advances in Bioinformatics and Computational Biology: Brazilian Symposium on Bioinformatics, BSB 2005, São Leopoldo, Brazil, volume 3594 of Lecture Notes in Bioinformatics, pages 42-53. Springer-Verlag, 2005.

[29] A. C. Lorena and A. C. P. L. F. Carvalho. Evolutionary design of multiclass support vector machines. Journal of Intelligent and Fuzzy Systems, 18:445-454, 2007.

[30] A. C. Lorena and A. C. P. L. F. Carvalho. Protein cellular localization prediction with multiclass support vector machines and decision trees. Computers in Biology and Medicine, 37(2):115-125, 2007.

[31] E. Mayoraz and E. Alpaydim. Support vector machines for multi-class classification. Research Report IDIAP-RR-98-06, Dalle Molle Institute for Perceptual Artificial Intelligence, Martigny, Switzerland, Maio 1998. 
[32] E. Mayoraz and M. Moreira. On the decomposition of polychotomies into dichotomies. Research Report 96-08, IDIAP, Dalle Molle Institute for Perceptive Artificial Intelligence, Martigny, Valais, Switzerland, December 1996.

[33] M. Mitchell. An introduction to Genetic Algorithms. MIT Press, 1999.

[34] T. Mitchell. Machine Learning. McGraw Hill, 1997.

[35] M. Moreira and E. Mayoraz. Improved pairwise coupling classification with correcting classifiers. Research report IDIAP-RR 97-09, IDIAP, Dalle Molle Institute of Perceptual Artificial Intelligence, Martigny, Switzerland, October 1997.

[36] A. Passerini. Kernel Methods, multiclass classification and applications to computational molecular biology. PhD thesis, Università Degli Studi di Firenze, 2004.

[37] A. Passerini, M. Pontil, and P. Frasconi. New results on error correcting output codes of kernel machines. IEEE Transactions on Neural Networks, 15:45-54, 2004.

[38] Thimaporn Phetkaew, Boonserm Kijsirikul, and Wanchai Rivepiboon. Learning multiclass support vector machines by reordering adaptive directed acyclic graph. In Proceedings of the 7th National Computer Science and Engineering Conference at Burapha University, 2003.

[39] Thimaporn Phetkaew, Boonserm Kijsirikul, and Wanchai Rivepiboon. Reordering adaptive directed acyclic graphs: an improved algorithm for multiclass support vector machines. In Proceedings of the International Conference on Neural Networks, pages 1605-1610. IEEE Computer Society Press, 2003.

[40] E. M. C. Pimenta. Abordagens para decomposição de problemas multiclasse: os códigos de correção de erros de saída. Master's thesis, Departamento de Ciências de Computadores, Faculdade de Ciências da Universidade do Porto, 2005.

[41] J. C. Platt, N. Cristiani, and J. Shawe-Taylor. Large margin DAGs for multiclass classification. In Advances in Neural Information Processing Systems, volume 12, pages 547-553. The MIT Press, 2000.

[42] J. Ross Quinlan. Induction of decision trees. Machine Learning, 1(1):81-106, 1986.

[43] R. Rifkin and A. Klautau. In defense of one-vs-all classification. Journal of Machine Learning Research, 5:1533-7928, 2004.

[44] G. Rätsch, A. J. Smola, and S. Mika. Adapting codes and embeddings for polychotomies. In Advances in Neural Information Processing Systems, volume 15, pages 513520. MIT Press, 2003. 
[45] P. Savicky and J. Fürnkranz. Combining pairwise classifiers with stacking. In Michael R. Berthold, Hans-Joachim Lenz, Elizabeth Bradley, Rudolf Kruse, and Christian Borgelt, editors, Advances in Intelligent Data Analysis V, 5th International Symposium on Intelligent Data Analysis, IDA 2003, pages 219-229, 2003.

[46] F. Schwenker. Hierarquical support vector machines for multi-class pattern recognition. In Proceedings of the 4th International Conference on Knowledge-Based Intelligent Systems and Allied Technologies, pages 561-565. IEEE Computer Society Press, 2000.

[47] F. Schwenker and G. Palm. Tree-structured support vector machines for multi-class pattern recognition. In J. Kittler and F. Roli, editors, Proceedings of the International Workshop on Multiple Classifier Systems, volume 2096 of Lecture Notes in Computer Science, pages 409-417. Springer-Verlag, 2001.

[48] A. J. Smola and B. Schölkopf. Learning with Kernels. The MIT Press, Cambridge, MA, 2002.

[49] F. Takahashi and S. Abe. Decision-tree-based multiclass support vector machines. In Proceedings of the 9th International Conference on Neural Information Processing, volume 3, pages 1418-1422, 2002.

[50] F. Takahashi and S. Abe. Optimizing directed acyclic graph support vector machines. In Proceedings of Artificial Neural Networks in Pattern Recognition, pages 166-170, 2003.

[51] V. N. Vapnik. The nature of Statistical learning theory. Springer-Verlag, New York, 1995.

[52] V. Vural and J. G. Dy. A hierarchical method for multi-class support vector machines. In Proceedings of the 21st International Conference on Machine Learning, pages 831838, Banff, Alberta, Canada, 2004.

[53] T. Windeatt and R. Ghaderi. Coding and decoding strategies for multi-class learning problems. Information Fusion, 4(1):11-21, 2003.

[54] E. Zitzler, M. Laumanns, and L. Thiele. SPEA2: Improving the strength pareto evolutionary algorithm. In Evolutionary Methods for Design, Optimisation, and Control, CIMNE, Barcelona, Spain, pages 95-100, 2002. 